\title{
Relevance of $\mathrm{hMLH1}-93 \mathrm{G}>\mathrm{A}, 655 \mathrm{~A}>\mathrm{G}$ and $1151 \mathrm{~T}>\mathrm{A}$ polymorphisms with colorectal cancer susceptibility: a meta-analysis based on 38 case-control studies
}

\author{
Mohammad Zare ${ }^{1}$ \\ (iD) Jamal Jafari-Nedooshan ${ }^{1}$ \\ Mohammadali Jafari ${ }^{2}$ \\ Hossein Neamatzadeh ${ }^{3}$ \\ Seyed Mojtaba Abolbaghaei4 \\ Elnaz Foroughi 5 \\ Rezvan Nasiri 6 \\ Masoud Zare-Shehneh ${ }^{3}$
}

\begin{abstract}
1. Department of General Surgery, Shahid Sadoughi University of Medical Sciences, Yazd, Iran 2. Department of Emergency Medicine, Shahid Sadoughi University of Medical Sciences, Yazd, Iran 3. Department of Medical Genetics, Shahid Sadoughi University of Medical Sciences, Yazd, Iran 4. Department of Forensic Medicine, Shahid Beheshti University of Medical Sciences, Tehran, Iran 5. Department of Pediatric Dentistry, Arak university of Medical Sciences, Arak, Iran 6. Department of Oral and Maxillofacial Surgery, Tehran University of Medical Sciences, Tehran, Iran
\end{abstract}

http://dx.doi.org/10.1590/1806-9282.64.10.942

\section{SUMMARY}

OBJECTIVE: There has been increasing interest in the study of the association between human mutL homolog 1 (hMLH1) gene polymorphisms and risk of colorectal cancer (CRC). However, results from previous studies are inconclusive. Thus, a meta-analysis was conducted to derive a more precise estimation of the effects of this gene.

METHODS: A comprehensive search was conducted in the PubMed, EMBASE, Chinese Biomedical Literature databases until January 1 , 2018. Odds ratio (OR) with 95\% confidence interval (CI) was used to assess the strength of the association.

RESULTS: Finally, 38 case-control studies in 32 publications were identified met our inclusion criteria. There were 14 studies with 20668 cases and 19533 controls on hMLH1 -93G>A, 11 studies with 5,786 cases and 8,867 controls on 655A>G and 5 studies with 1409 cases and 1637 controls on 1151T>A polymorphism. The combined results showed that $655 \mathrm{~A}>\mathrm{G}$ and $1151 T>A$ polymorphisms were signifcantly associated with CRC risk, whereas $-93 \mathrm{G}>A$ polymorphism was not significantly associated with CRC risk. As for ethnicity, $-93 G>A$ and $655 A>G$ polymorphisms were associated with increased risk of CRC among Asians, but not among Caucasians. More interestingly, subgroup analysis indicated that $655 \mathrm{~A}>\mathrm{G}$ might raise $C R C$ risk in PCR-RFLP and HB subgroups.

CONCLUSION: Inconsistent with previous meta-analyses, this meta-analysis shows that the hMLH1 655A>G and 1151T>A polymorphisms might be risk factors for CRC. Moreover, the -93G>A polymorphism is associated with the susceptibility of CRC in Asian population.

KEYWORDS: colorectal cancer, hMLH1, polymorphism; Meta-analysis.

DATE OF SUBMISSION: 19-Jan-2018

DATE OF ACCEPTANCE: 24-Mar-2018

CORRESPONDING AUTHOR: Jamal Jafari-Nedooshan - University of Medical Sciences, Yazd, Iran

Shohadaye Gomnam - Yazd - Yazd - Iran - 0098335 


\section{INTRODUCTION}

Colorectal cancer (CRC) is one of the most frequent malignant tumours of the digestive tract in human, especially in the Western world., ${ }^{1,2}$ CRC ranks among the three most common cancers in terms of both cancer incidence and cancer-related deaths in most developed countries. More than one million cases of CRC are diagnosed in the worldwide every year. ${ }^{1-3}$ The cross cultural and migrant studies suggest that the majority of CRC cases $(\approx 85 \%)$ is related to environmental factors including smoking, drinking, meat consumption, less activity, exposure to aryl amines, and heterocyclic amines. ${ }^{4-6}$

The MMR genes encode a family of highly conserved proteins, including MLH1, MSH2, MSH6, and PMS2 ${ }^{7,8}$ MMR systems promote genetic stability by repairing DNA replication errors, inhibiting recombination between non-identical DNA sequences, and participating in responses to DNA damage. ${ }^{9,10}$ MLH1 protein physically interacts with other MMR components; although the exact role of MLH1 gene remains elusive, MLH1-deficiency is associated with cancer predisposition. ${ }^{11}$ To date, most of the causative mutations have been identified in MLH1 gene. Mutations in the gene for MLH1 are estimated to account for nearly $40 \%$ of the more than 400 known MMR gene mutations, and prevalence of mutations in MLH1 in Western countries is between 1 of $1000 .{ }^{12}$ Standardized incidence ratios (SIRs) for carriers of hMLH1 mutations, when compared with the general population is 68 and the relative risk for CRC for first-degree relatives of mutation carriers compared with first degree relatives of non-carriers is 8.1. ${ }^{13,14}$

Molecular epidemiological and pooling analyses studies have reported the association of hMLH1 $-93 \mathrm{G}>\mathrm{A}, 655 \mathrm{~A}>\mathrm{G}$ and $1151 \mathrm{~T}>\mathrm{A}$ with $\mathrm{CRC}$ risk, ${ }^{15-46}$ but the results remain inconsistent and inclusive. Inconsistencies in results may be caused by differences in study design, population, or different statistical methods. Meta-analysis is a powerful tool for summarizing the different studies. It can not only overcome the problem of small size and inadequate statistical power of genetic studies of complex traits, but also provide more reliable results than a single case-control study. However, the previous meta-analysis on hMLH1 polymorphisms with CRC risk has shown conflicting conclusions. Because, several published studies were not included in the meta-analysis and additional original studies with larger sample sizes have been published since then. We therefore performed a meta-analysis to make a more precise assessment the association between hMLH1 polymorphisms with CRC risk, by adding more studies implemented in recent years.

\section{METHODS}

Search Strategy

A comprehensive literature search was performed using the MEDLINE (National Library of Medicine), EMBASE (Excerpta Medica), CANCERLIT (National Cancer Institute), Web of Science (Thomson-Reuters), Cochrane Library, Chinese Biomedical Literature Database and Google scholar for all relevant articles published up to January 1, 2018 that evaluated the association between the hMLH1 gene polymorphisms and risk of CRC. The following terms were included in the search: "Colorectal cancer" or "CRC", "human mutL homolog 1" or "hMLH1",'-93G>A" or "rs1800734", "655A>G” or "p. Ile219Val" or "rs1799977", "1151T>A" or "p.Val384Asp" or "rs63750447", "polymorphism", "mutation", "variant", "gene", "genotype", "SNP”, and "allele". The search was not restricted by the publication year or language. Furthermore, in order to identify potentially relevant studies, we manually searched reference lists of eligible studies, reviews and related meta-analyses. If there were multiple reports of the same sample or overlapping data only the study with the largest sample sizes or the most recent one was included following careful examination.

\section{Inclusion and Exclusion Criteria}

Studies were selected according to the following inclusion criteria: (1) full-text published studies; (2) epidemiological studies with case-control or cohort design; (3) investigating the association between hMLH1 polymorphisms and CRC risk; (4) providing sufficient genotype data or information that could help infer the results in the studies to calculate the odd ratios (ORs) with a 95\% confidence interval (CI). The exclusion criteria were as follows: (1) studies with only case population (no control population); (2) studies without detail genotype frequencies, which were unable to calculate odds ratio; (3) duplicate of previous publication.

\section{Data Extraction}

Information was carefully extracted from all eligible studies independently by two investigators ac- 
cording to the inclusion criteria. For each study the following information was extracted: name of first author, publication year, country of origin, ethnicity, polymorphisms, source of controls, genotyping method, number of cases and controls, genotype frequency in cases and controls, minor allele frequencies (MAFs) in control subjects, and Hardy-Weinberg equilibrium test in control subjects. Diverse ethnicities were categorized as Caucasian, Asian, African and Mixed, which included more than one race. Disagreements were resolved in consultation with the third reviewer.

\section{STATISTICAL ANALYSIS}

The strength of associations was assessed by using odds ratios (ORs) and 95\% confidence interval (CIs). The significance of the pooled OR was determined by the Z-test; a P value of $<0.05$ was considered significant. The OR of hMLH1 polymorphisms and CRC risk was estimated for each study. The pooled ORs were performed for allele (B vs. A), homozygote (BB vs. $\mathrm{AA})$, heterozygote (AB vs. $\mathrm{AA})$, dominant (BB+$\mathrm{BA}$ vs. $\mathrm{AA}$ ) and recessive (BB vs. $\mathrm{AB}+\mathrm{AA}$ ) models. $\mathrm{A}$ Chi square-test based Q-statistic test and an $\mathrm{I}^{2}$ statistics $\left(\mathrm{I}^{2}=100 \% \times(\mathrm{Q}-\mathrm{df}) / \mathrm{Q}\right)$ were performed to assess the heterogeneity between studies. ${ }^{47}$ A significant Q-statistic $(\mathrm{P}<0.10)$ indicated heterogeneity across studies. Venice criteria for the $\mathrm{I}^{2}$ statistics: " $\mathrm{I}^{2}<25 \%$ represents no heterogeneity, $\mathrm{I}^{2}=25-50 \%$ represents moderate heterogeneity, $\mathrm{I}^{2}=50-75 \%$ represents large heterogeneity and $\mathrm{I}^{2}>75 \%$ represents extreme heterogeneity". Dependent on the results of heterogeneity test among individual studies, the fixed effect model (Mantel-Haenszel method) or random effect model (DerSimonian-Laird method) was utilized to summarize the pooled OR. ${ }^{1,48}$ Furthermore, to detect the source of between-study heterogeneity, subgroup analyses (Meta-regression) by ethnicity, genotyping method and source of controls were performed. A Hardy-Weinberg equilibrium (HWE) was assessed for each study using the goodness-of-fit test (Chi square-test or Fisher exact test) only in control groups, and deviation was considered when $\mathrm{P}<0.01$. The one-way sensitivity analyses were performed to survey the stability of the results, namely, a single study in the meta-analysis was omitted each time to reflect the influence of the individual data set to the pooled OR. Publication bias was evaluated by visual inspection of the funnel plot and Egger's linear regression test, and the significance level was set at 0.05 for both. ${ }^{49,50}$ If publication bias observed, the Duval and Tweedie non-parametric "trim and fill" method was assessed to adjust for it. All the statistical analyses were performed by Comprehensive Meta-Analysis (CMA) software (Version 2.20; Biostat, USA). $\mathrm{P}<0.05$ (two-tailed) was considered statistically significant.

\section{RESULTS}

\section{Study Selection and Characteristics}

After deleting of duplicates, 361 articles were excluded from screening the titles and abstracts, as these were unrelated to hMLH1 polymorphisms, or CRC risk. Further, 40 articles was excluded for no genotypic information, reviews, letters, case report, clinical, and animal studies. Totally, 38 case-control studies in 32 publications ${ }^{15-46}$ containing 4092 cases and 5909 controls were included in the meta-analysis. The studies were published from 1998 to 2017. The main characteristics of the selected studies and the genotype distribution of the hMLH1 gene polymorphisms are summarized in Table 1. Of the 30 studies, eight were conducted in Asians (Japan, Korea, China, Kazakhstan, Malaysia, and Iran), 5 in Caucasians (Canada, Czech, USA, UK, Spain, Denmark, and Sweden), and one in a mixed population (Mexico). Of them, there were 19 studies with 20,668 CRC cases and 19,533 controls for $-93 \mathrm{G}>\mathrm{A}$ (rs1800734) polymorphism, eleven studies with 5,786 CRC cases and 8,867 controls for hMLH1 655A>G (rs1799977) polymorphism, and eight studies with 1,409 CRC cases and 1,637 controls for hMLH1 1151T>A (rs63750447) polymorphism. For the ethnicities, 12 studies of Caucasians and six studies of Asians were included on the hMLH1 -93G >A (rs1800734). As to hMLH1 $655 \mathrm{~A}>\mathrm{G}$ (rs1799977) polymorphism, six studies of Caucasians and four studies of Asians were included. The eight studies on hMLH1 1151T>A (rs63750447) polymorphism were all based on the Asians. The distribution of the genotypes in the control subjects was in agreement with HWE except three studies.

\section{Quantitative Synthesis}

hMLH1 -93G>A (rs1800734) Polymorphism

The main results of the meta-analysis for all 19 case-control studies ${ }^{15-33}$ on hMLH1 -93G $>$ A polymorphism are presented in Table 3. The results of pooling all studies showed that there was no statistically 
TABLE 1: MAIN CHARACTERISTICS OF STUDIES INCLUDED IN THIS META-ANALYSIS.

\begin{tabular}{|c|c|c|c|c|c|c|c|c|c|c|c|c|c|c|c|c|}
\hline \multirow{3}{*}{$\begin{array}{l}\text { First Author } \\
-93 G>A(r s 1800734)\end{array}$} & \multirow{3}{*}{$\begin{array}{l}\text { Country } \\
\text { (Ethnicity) }\end{array}$} & \multirow[t]{3}{*}{ SOC } & \multirow{3}{*}{$\begin{array}{l}\text { Genotyping } \\
\text { Technique }\end{array}$} & \multirow[t]{3}{*}{ Case/Control } & \multicolumn{5}{|l|}{ Cases } & \multicolumn{5}{|c|}{ Controls } & \multirow[t]{3}{*}{ MAFs } & \multirow[t]{3}{*}{ HWE } \\
\hline & & & & & \multicolumn{3}{|c|}{ Genotypes } & \multicolumn{2}{|l|}{ Allele } & \multicolumn{3}{|c|}{ Genotypes } & \multicolumn{2}{|l|}{ Allele } & & \\
\hline & & & & & GG & AG & AA & G & $A$ & GG & AG & $\mathrm{AA}$ & G & A & & \\
\hline Ito 199915 & Japan (Asian) & PB & $\begin{array}{l}\text { PCR- } \\
\text { SSCP }\end{array}$ & $27 / 84$ & 8 & 10 & 9 & 26 & 28 & 22 & 46 & 16 & 90 & 78 & 0.464 & 0.355 \\
\hline Shin 200216 & Korea (Asian) & $\mathrm{HB}$ & $\begin{array}{l}\text { PCR- } \\
\text { SSCP }\end{array}$ & 139/157 & 33 & 61 & 45 & 127 & 151 & 42 & 74 & 41 & 158 & 156 & 0.496 & 0.472 \\
\hline Raptis 200717 & Canada (Caucasian) & PB & TaqMan & 929/1098 & 554 & 331 & 44 & 1439 & 419 & 687 & 352 & 59 & 1726 & 470 & 0.214 & 0.118 \\
\hline Chen 200718 & USA(Caucasian) & NA & Pyroseq & $99 / 286$ & 44 & 47 & 8 & 135 & 63 & 169 & 99 & 18 & 437 & 135 & 0.236 & 0.497 \\
\hline Tulupova 200819 & Czech (Caucasian) & $\mathrm{HB}$ & TaqMan & $619 / 611$ & 359 & 216 & 44 & 934 & 304 & 365 & 209 & 37 & 939 & 283 & 0.231 & 0.336 \\
\hline Samowitz 200820 & USA(Caucasian) & PB & DS & 1006/1963 & 610 & 344 & 52 & 1564 & 448 & 1170 & 688 & 105 & 3028 & 898 & 0.228 & 0.768 \\
\hline Koessler 200821 & UK(Caucasian) & PB & TaqMan & $2288 / 2276$ & 1407 & 778 & 103 & 3592 & 984 & 1392 & 777 & 107 & 3561 & 991 & 0.217 & 0.914 \\
\hline Allan 200822 & UK(Caucasian) & NA & PCR-RFLP & $1518 / 589$ & 878 & 566 & 74 & 2322 & 714 & 369 & 196 & 24 & 934 & 244 & 0.207 & 0.750 \\
\hline Campbell 200923 & USA(Caucasian) & PB & PCR-RFLP & $1600 / 1963$ & 952 & 553 & 95 & 2457 & 743 & 1170 & 688 & 105 & 3028 & 898 & 0.228 & 0.768 \\
\hline van Roon 201024 & $\begin{array}{l}\text { Netherland(Cau- } \\
\text { casian) }\end{array}$ & NA & DS & $39 / 920$ & 12 & 20 & 7 & 44 & 34 & 554 & 331 & 44 & 1425 & 415 & 0.225 & 0.542 \\
\hline Whiffin 201125 & UK(Caucasian) & PB & KASPae & $10409 / 6965$ & 6408 & 3504 & 497 & 16320 & 4498 & 4395 & 2261 & 309 & 11051 & 2879 & 0.206 & 0.401 \\
\hline Savio 201226 & Canada (Caucasian) & PB & PCR-RFLP & $252 / 845$ & 150 & 96 & 6 & 396 & 108 & 528 & 264 & 53 & 1320 & 370 & 0.218 & 0.011 \\
\hline $\begin{array}{l}\text { Muniz-Mendoza } \\
201227\end{array}$ & Mexico (Mixed) & $\mathrm{HB}$ & PCR-RFLP & $100 / 115$ & 47 & 44 & 9 & 138 & 62 & 39 & 55 & 21 & 133 & 97 & 0.421 & 0.834 \\
\hline Nizam 201328 & Malaysia (Asian) & $\mathrm{HB}$ & PCR-RFLP & $104 / 104$ & 22 & 50 & 32 & 94 & 114 & 33 & 33 & 38 & 99 & 109 & 0.524 & 0.520 \\
\hline $\begin{array}{l}\text { Martinez-Uruena } \\
201329\end{array}$ & Spain (Caucasian) & $\mathrm{HB}$ & PCR-RFLP & $383 / 236$ & 233 & 131 & 19 & 597 & 169 & 129 & 102 & 5 & 360 & 112 & 0.237 & 0.002 \\
\hline $\begin{array}{l}\text { Djansugurova } 2015 \\
30\end{array}$ & Kazakhstan (Asian) & $\mathrm{HB}$ & PCR-RFLP & $249 / 244$ & 126 & 94 & 29 & 346 & 152 & 101 & 115 & 28 & 317 & 171 & 0.350 & 0.581 \\
\hline Li 201531 & China(Asian) & NA & PCR-RFLP & $451 / 629$ & 88 & 198 & 165 & 374 & 528 & 218 & 301 & 110 & 737 & 521 & 0.414 & 0.728 \\
\hline Zhang 201632 & China(Asian) & $\mathrm{HB}$ & TaqMan & $312 / 300$ & 66 & 139 & 107 & 271 & 353 & 52 & 154 & 94 & 258 & 342 & 0.570 & 0.413 \\
\hline Mik 201733 & Poland (Caucasian) & NA & PCR-RFLP & 144/151 & 74 & 45 & 25 & 193 & 95 & 53 & 61 & 37 & 167 & 135 & 0.447 & 0.024 \\
\hline 655A>G(rs1799977) & & & & 20668/19533 & AA & $A G$ & GG & A & G & AA & AG & GG & A & G & & \\
\hline Kim 200434 & Korea (Asian) & PB & TaqMan & $107 / 330$ & 100 & 7 & 0 & 207 & 7 & 311 & 18 & 1 & 640 & 20 & 0.030 & 0.191 \\
\hline Mei 200635 & China (Asian) & $\mathrm{HB}$ & PCR & $160 / 150$ & 144 & 14 & 2 & 302 & 18 & 141 & 9 & 0 & 291 & 9 & 0.030 & 0.704 \\
\hline Raptis 200717 & Canada (Caucasian) & PB & TaqMan & 929/1098 & 451 & 391 & 87 & 1293 & 565 & 514 & 485 & 99 & 1513 & 683 & 0.311 & 0.309 \\
\hline Berndt 200736 & USA(Caucasian) & PB & TaqMan & $211 / 2090$ & 100 & 94 & 17 & 294 & 128 & 968 & 896 & 226 & 2832 & 1348 & 0.322 & 0.386 \\
\hline Christensen 200837 & Denmark(Caucasian) & PB & SBE-tags & $380 / 770$ & 172 & 170 & 38 & 514 & 246 & 364 & 327 & 79 & 1055 & 485 & 0.314 & 0.660 \\
\hline Nejda 200938 & Spain (Caucasian) & $\mathrm{HB}$ & PCR-RFLP & $140 / 125$ & 41 & 72 & 27 & 154 & 126 & 64 & 44 & 17 & 172 & 78 & 0.312 & 0.044 \\
\hline Campbell 200923 & USA(Caucasian) & PB & PCR-RFLP & $1601 / 1944$ & 764 & 678 & 159 & 2206 & 996 & 937 & 848 & 159 & 2722 & 1166 & 0.299 & 0.087 \\
\hline Picelli 201039 & Sweden (Caucasian) & PB & DS & $1781 / 1701$ & 819 & 781 & 181 & 2419 & 114 & 832 & 708 & 161 & 2372 & 1030 & 0.302 & 0.636 \\
\hline $\begin{array}{l}\text { Muniz-Mendoza } \\
201227\end{array}$ & Mexico (Mixed) & $\mathrm{HB}$ & PCR-RFLP & $102 / 100$ & 71 & 26 & 5 & 168 & 36 & 81 & 19 & 0 & 181 & 19 & 0.095 & 0.293 \\
\hline Milanizadeh 201340 & Iran (Asian) & $\mathrm{HB}$ & PCR-RFLP & $219 / 248$ & 25 & 62 & 132 & 112 & 326 & 248 & 54 & 119 & 227 & 269 & 0.346 & $\leq 0.001$ \\
\hline Peng 201641 & China (Asian) & PB & PCR-HRM & $156 / 311$ & 151 & 5 & 0 & 151 & 5 & 307 & 4 & 0 & 618 & 4 & 0.006 & 0.909 \\
\hline $1151 T>A(r s 63750447)$ & & & & $5786 / 8867$ & TT & AT & AA & $\mathrm{T}$ & A & $\mathrm{TT}$ & AT & AA & $\mathrm{T}$ & A & & \\
\hline Wang 199842 & China (Asian) & NA & $\begin{array}{l}\text { PCR- } \\
\text { SSCP }\end{array}$ & $26 / 80$ & 22 & 4 & 0 & 48 & 4 & 77 & 3 & 0 & 157 & 3 & 0.018 & 0.864 \\
\hline Wang 200043 & China (Asian) & $\mathrm{HB}$ & $\begin{array}{l}\text { PCR- } \\
\text { SSCP }\end{array}$ & $101 / 100$ & 88 & 13 & 0 & 189 & 13 & 94 & 6 & 0 & 194 & 6 & 0.030 & 0.757 \\
\hline Kim 200434 & Korea (Asian) & PB & TaqMan & $107 / 330$ & 100 & 7 & 0 & 207 & 7 & 313 & 17 & 0 & 643 & 17 & 0.025 & 0.631 \\
\hline Zhang 200544 & China (Asian) & PB & DHPLC & $90 / 268$ & 82 & 8 & 0 & 172 & 8 & 251 & 17 & 0 & 519 & 17 & 0.031 & 0.591 \\
\hline Mei 200635 & China (Asian) & $\mathrm{HB}$ & PCR & $160 / 150$ & 142 & 18 & 0 & 302 & 18 & 141 & 9 & 0 & 291 & 9 & 0.030 & 0.474 \\
\hline Ohsawa 200945 & Japan (Asian) & NA & PCR-RFLP & $670 / 332$ & 630 & 39 & 1 & 1299 & 41 & 327 & 5 & 0 & 659 & 5 & 0.007 & 0.890 \\
\hline Wang 201046 & China (Asian) & NA & DHPLC & $99 / 66$ & 83 & 16 & 0 & 182 & 16 & 63 & 3 & 0 & 129 & 3 & 0.022 & 0.850 \\
\hline Peng 201641 & China (Asian) & PB & PCR-HRM & $156 / 311$ & 142 & 13 & 1 & 297 & 15 & 310 & 1 & 0 & 621 & 1 & 0.001 & 0.977 \\
\hline
\end{tabular}

SOP, source of population; HB, Hospital-based study; PB, Population-based study; RT-PCR, Real-Time PCR; PCR-RFLP, PCR-restriction fragment length polymorphism; MALDI-TOF MS, matrix-assisted laser desorption/ionization time-of-flight mass spectrometry; MAFs, Minor Allele Frequencies; HWE, Hardy-Weinberg equilibrium; SBE-tags: Single base extension; HRM : High Resolution Melting. 
TABLE 2. THE META-ANALYSIS OF HMLH1 -93G>A (RS1800734) POLYMORPHISMS AND CRC RISK.

\begin{tabular}{|c|c|c|c|c|c|c|c|c|c|c|}
\hline \multirow[t]{2}{*}{ Subgroup } & \multirow[t]{2}{*}{ Genetic Model } & \multirow[t]{2}{*}{ Type of Model } & \multicolumn{2}{|c|}{ Heterogeneity } & \multicolumn{4}{|c|}{ Odds Ratio } & \multicolumn{2}{|c|}{ Publication Bias } \\
\hline & & & $12(\%)$ & $\mathrm{PH}$ & OR & $95 \% \mathrm{Cl}$ & Ztest & POR & PBeggs & PEggers \\
\hline \multirow[t]{5}{*}{ Overall $(n=19)$} & A vs. G & Random & 99.00 & $\leq 0.001$ & 0.946 & $0.650-1.379$ & -0.287 & 0.774 & 0.161 & 0.936 \\
\hline & AA vs. GG & Random & 80.83 & $\leq 0.001$ & 1.220 & $0.950-1.566$ & 1.555 & 0.120 & 0.363 & 0.769 \\
\hline & AG vs. GG & Random & 98.69 & $\leq 0.001$ & 0.914 & $0.546-1.532$ & -0.341 & 0.733 & 0.141 & 0.625 \\
\hline & $A A+A G$ vs. $G G$ & Random & 75.86 & $\leq 0.001$ & 1.066 & 0.954-1.191 & 1.127 & 0.260 & 1.000 & 0.838 \\
\hline & $A A$ vs. $A G+G G$ & Random & 80.60 & $\leq 0.001$ & 1.191 & $0.954-1.486$ & 1.547 & 0.122 & 0.401 & 0.715 \\
\hline \multicolumn{11}{|l|}{ By ethnicity } \\
\hline \multirow{5}{*}{$\begin{array}{l}\text { Caucasians } \\
(n=12)\end{array}$} & A vs. G & Random & 99.35 & $\leq 0.001$ & 0.882 & $0.535-1.453$ & -0.492 & 0.623 & 0.303 & 0.875 \\
\hline & AA vs. GG & Random & 66.00 & 0.001 & 1.061 & $0.859-1.310$ & 0.549 & 0.583 & 0.192 & 0.716 \\
\hline & AG vs. GG & Random & 99.16 & $\leq 0.001$ & 0.826 & $0.422-1.619$ & -0.556 & 0.578 & 0.064 & 0.807 \\
\hline & $A A+A G$ vs. $G G$ & Random & 68.02 & $\leq 0.001$ & 1.056 & $0.958-1.164$ & 1.089 & 0.276 & 0.631 & 0.703 \\
\hline & $A A$ vs. $A G+G G$ & Random & 53.03 & 0.019 & 1.090 & $0.911-1.304$ & 0.943 & 0.346 & 0.119 & 0.516 \\
\hline \multirow[t]{5}{*}{ Asians $(n=6)$} & A vs. G & Random & 88.14 & $\leq 0.001$ & 1.179 & $0.840-1.655$ & 0.951 & 0.342 & 1.000 & 0.355 \\
\hline & AA vs. GG & Random & 80.19 & $\leq 0.001$ & 1.759 & $1.054-2.934$ & 2.163 & 0.031 & 0.452 & 0.102 \\
\hline & AG vs. GG & Random & 88.07 & $\leq 0.001$ & 1.168 & $0.644-2.119$ & 0.513 & 0.608 & 0.707 & 0.795 \\
\hline & $A A+A G$ vs. $G G$ & Random & 84.60 & $\leq 0.001$ & 1.139 & $0.708-1.833$ & 0.535 & 0.592 & 1.000 & 0.593 \\
\hline & $A A$ vs. $A G+G G$ & Random & 81.46 & $\leq 0.001$ & 1.381 & $0.885-2.155$ & 1.421 & 0.155 & 0.707 & 0.330 \\
\hline \multicolumn{11}{|l|}{$\begin{array}{l}\text { By Popula- } \\
\text { tion-Based }\end{array}$} \\
\hline \multirow{5}{*}{ PB $(n=7)$} & A vs. G & Random & 99.63 & $\leq 0.001$ & 0.720 & $0.349-1.486$ & -0.888 & 0.375 & 0.071 & 0.640 \\
\hline & AA vs. GG & Fixed & 18.16 & 0.291 & 1.020 & $0.917-1.136$ & 0.368 & 0.713 & 0.548 & 0.168 \\
\hline & AG vs. GG & Random & 99.51 & $\leq 0.001$ & 0.600 & $0.221-1.628$ & -1.004 & 0.316 & 0.071 & 0.863 \\
\hline & $A A+A G$ vs. $G G$ & Fixed & 0.00 & 0.656 & 1.042 & 0.995-1.092 & 1.753 & 0.080 & 1.000 & 0.578 \\
\hline & $A A$ vs. $A G+G G$ & Fixed & 37.36 & 0.143 & 1.030 & $0.927-1.145$ & 0.558 & 0.577 & 0.548 & 0.446 \\
\hline \multirow{5}{*}{$\mathrm{HB}(n=7)$} & A vs. G & Fixed & 41.77 & 0.112 & 0.971 & $0.878-1.074$ & -0.573 & 0.566 & 0.548 & 0.392 \\
\hline & AA vs. GG & Random & 78.04 & $\leq 0.001$ & 1.526 & $0.963-2.418$ & 1.800 & 0.072 & 0.229 & 0.101 \\
\hline & AG vs. GG & Random & 81.22 & $\leq 0.001$ & 0.943 & $0.650-1.368$ & -0.308 & 0.758 & 0.367 & 0.477 \\
\hline & $A A+A G$ vs. $G G$ & Random & 55.17 & 0.037 & 0.890 & $0.711-1.114$ & -1.017 & 0.309 & 1.000 & 0.882 \\
\hline & $A A$ vs. $A G+G G$ & Fixed & 35.45 & 0.158 & 1.087 & $0.895-1.321$ & 0.840 & 0.401 & 0.763 & 0.763 \\
\hline \multicolumn{11}{|c|}{ By Genotyping Technique } \\
\hline \multirow{5}{*}{$\begin{array}{l}\text { PCR-RFLP } \\
(n=9)\end{array}$} & Tvs. C & Random & 89.42 & $\leq 0.001$ & 0.984 & $0.784-1.234$ & -0.141 & 0.888 & 0.251 & 0.306 \\
\hline & AA vs. GG & Random & 87.93 & $\leq 0.001$ & 0.999 & $0.592-1.686$ & -0.004 & 0.997 & 0.602 & 0.232 \\
\hline & AG vs. GG & Random & 85.77 & $\leq 0.001$ & 1.031 & $0.782-1.360$ & 0.217 & 0.828 & 0.916 & 0.950 \\
\hline & $A A+A G$ vs. $G G$ & Random & 84.94 & $\leq 0.001$ & 0.997 & $0.775-1.284$ & -0.022 & 0.983 & 0.251 & 0.624 \\
\hline & $A A$ vs. $A G+G G$ & Random & 88.95 & $\leq 0.001$ & 1.146 & 0.693-1.896 & 0.531 & 0.595 & 0.117 & 0.384 \\
\hline \multirow[t]{5}{*}{$\operatorname{TaqMan}(n=4)$} & A vs. G & Fixed & 0.00 & 0.720 & 1.017 & 0.947-1.093 & 0.467 & 0.641 & 1.000 & 0.513 \\
\hline & AA vs. GG & Random & 84.67 & $\leq 0.001$ & 1.291 & $0.795-2.097$ & 1.034 & 0.301 & 0.734 & 0.585 \\
\hline & AG vs. GG & Fixed & 39.21 & 0.177 & 1.024 & $0.934-1.124$ & 0.506 & 0.613 & 0.734 & 0.635 \\
\hline & $A A+A G$ vs. $G G$ & Fixed & 13.49 & 0.325 & 1.022 & $0.935-1.117$ & 0.475 & 0.635 & 0.734 & 0.762 \\
\hline & $A A$ vs. $A G+G G$ & Fixed & 0.00 & 0.649 & 1.018 & $0.855-1.212$ & 0.203 & 0.839 & 1.000 & 0.680 \\
\hline
\end{tabular}

PCR-RFLP, PCR-restriction fragment length polymorphism; HWE, Hardy-Weinberg equilibrium.

significant association between hMLH1 -93G>A polymorphism and the risk of CRC.

To evaluate the potential effects of specific study characteristics on the association between hMLH1 -93G > A polymorphism and CRC risk; we pooled the ORs and 95\% CIs by the subgroups analysis of ethnicity, control source, and genotyping technique. When stratified by ethnicity, significant associa- tion between hMLH1 -93G>A polymorphism and CRC risk was detected among the Asian population under the homozygote model $(\mathrm{OR}=2.283,95 \% \mathrm{CI}$ 1.810-2.880, $\mathrm{P}<0.001$ ), but not among Caucasians. Furthermore, no significant associations were detected when the studies were stratified based on the source of control subjects and genotyping method (Table 2). 


\section{hMLH1 655A>G (rs1799977) Polymorphism}

The main results of the meta-analysis for all eleven case-control studies ${ }^{17,23,27,34-41}$ on hMLH1 $655 \mathrm{~A}>\mathrm{G}$ polymorphism are presented in Table 4 . The results of pooling all studies showed that there was a significant association between hMLH1 655A $>$ G polymorphism and the risk of CRC under the heterozygote ( $\mathrm{OR}=1.493,95 \%$ CI 1.147-1.944, $\mathrm{P}=0.865, \mathrm{P}=0.003)$, dominant $(\mathrm{OR}=1.298,95 \% \mathrm{CI} 1.085-1.553, \mathrm{P}=0.004)$ and recessive $(\mathrm{OR}=1.150,95 \% \mathrm{CI} 1.020-1.297, \mathrm{P}=$ 0.022) models.

In the subgroup analysis by ethnicity, we found a significant association between the hMLH1 $655 \mathrm{~A}>\mathrm{G}$ polymorphism and the risk of CRC in Asians under the allele (OR $=2.251,95 \%$ CI 1.758 $2.884, \mathrm{P}<0.001)$, homozygote $(\mathrm{OR}=10.262,95 \% \mathrm{CI}$ $6.419-16.405, \mathrm{P}<0.001)$, dominant $(\mathrm{OR}=2.411,95 \%$ CI 1.663-3.495, $\mathrm{P}<0.001)$ and recessive $(\mathrm{OR}=1.660$, 95\% CI 1.155-2.385, $\mathrm{P}<0.001)$ models with a fixed effect, whereas there was no significant association in any of the genetic models with a random effect models in Caucasians. When stratified by source of controls, significant association between hMLH1 $655 \mathrm{~A}>\mathrm{G}$ polymorphism and $\mathrm{CRC}$ risk was observed in hospital-based controls in the allele $(\mathrm{OR}=2.153$, 95\% CI 1.763-2.628, $\mathrm{P} \leq 0.001)$, homozygote ( $\mathrm{OR}=$ 5.873, 95\% CI 1.911-18.04, $\mathrm{P}=0.036)$, heterozygote $(\mathrm{OR}=2.955,95 \%$ CI 1.111-7.859, $\mathrm{P}=0.036)$, dominant $(\mathrm{OR}=2.513,95 \% \mathrm{CI} 1.876-3.367, \mathrm{P} \leq 0.001)$, and recessive $(\mathrm{OR}=1.671,95 \% \mathrm{CI} 1.216-2.297, \mathrm{P}=0.036)$ models, but not in the in population-based controls. Furthermore, hMLH1 $655 \mathrm{~A}>\mathrm{G}$ polymorphism was significantly associated with increased CRC risk in the subgroup of PCR-RFLP genotyping method in the allele model $(\mathrm{OR}=1.725,95 \%$ CI 1.038-2.866, $\mathrm{P}=0.036)$, dominant $(\mathrm{OR}=1.961,95 \%$ CI 0.999 3.847, $\mathrm{P}=0.05)$ and recessive $(\mathrm{OR}=1.366,95 \% \mathrm{CI}$ 1.133-1.647, $\mathrm{P}=0.001)$ models. In contrast, no significant association was observed in TaqMan genotyping subgroup (Table 3).

\section{hMLH1 1151T>A (rs63750447) Polymorphism}

The main results of the meta-analysis for all case-control eight studies ${ }^{34,35,41-46}$ on hMLH1 1151T $>$ A polymorphism are presented in Table 5 . Significant association between hMLH1 1151T $>$ A polymorphism and CRC was observed in the allele $(\mathrm{OR}=2.462,95 \%$ CI 1.763-2.628, $\mathrm{P} \leq 0.001$ ), homozygote ( $\mathrm{OR}=2.501$, 95\% CI 1.593-3.806, $\mathrm{P} \leq 0.001)$ and dominant $(\mathrm{OR}=$ 2.526, 95\% CI 1.622-3.934, P $\leq 0.001$ ) models (Table 3).

\section{Sensitivity analysis}

Sensitivity analysis was conducted by deleting each study in turn from the pooled analysis to examine the stability of the results. However, no individual study changed the pooled OR qualitatively, indicating that the pooled results were statistically robust.

\section{Publication bias}

We have assessed publication bias qualitatively by Begg's funnel plot and quantitatively by Egger's test. The shapes of the funnel plot did not indicate any evidence of obvious asymmetry in all genotypes in overall population. However, the results of Egger's test statistically confirmed the evidence of publication bias in the dominant model for hMLH1 655A $>\mathrm{G}$ polymorphism (PBeggs $=0.146$, PEggers $=0.021$ ). Therefore, we have used the Duval and Tweedie non-parametric "trim and fill" method to adjust for publication bias. However, meta-analysis with and without "trim and fill" did not draw different conclusion, indicating that our results were statistically robust. Moreover, neither Begg's funnel plot nor Egger's test detected obvious evidence of publication bias in subgroup analysis based on ethnicity, source of controls and genotyping methods by using of Begg's and Egger's test.

\section{DISCUSSION/CONCLUSION}

An increasing number of studies on genetic association studies, genome-wide association studies (GWASs), and relate meta-analyses have been published to clarify the association between gene polymorphisms and CRC..$^{32,33,41}$ Theoretically, polymorphisms in the hMLH1 gene could change the function of this gene, disturb the DNA repair and increase risk of CRC. ${ }^{32,33}$ The role of hMLH1 polymorphisms in the risk of $\mathrm{CRC}$ is controversial. The association between hMLH1 gene polymorphisms and risk of CRC has been a topic of particular interest, but the results from individual studies had been inconsistent and controversial. To better define the possible association, we carried out a comprehensive meta-analysis of hMLH1 polymorphisms.

Overall, our meta-analysis indicates that $655 \mathrm{~A}>\mathrm{G}$ and $1151 \mathrm{~T}>\mathrm{A}$ polymorphisms are associated with increased CRC risk when all eligible studies were pooled into the meta-analysis, whereas $-93 \mathrm{G}>\mathrm{A}$ polymorphism was not significantly associated with CRC risk. In further stratified, significantly increased 
TABLE 3. THE META-ANALYSIS OF HMLH1 655A>G AND 1151T>A POLYMORPHISMS AND CRC RISK.

\begin{tabular}{|c|c|c|c|c|c|c|c|c|c|c|}
\hline \multirow[t]{2}{*}{ subgroup } & \multirow[t]{2}{*}{ Genetic Model } & \multirow{2}{*}{$\begin{array}{l}\text { Type of } \\
\text { Model }\end{array}$} & \multicolumn{2}{|c|}{ Heterogeneity } & \multicolumn{4}{|c|}{ Odds Ratio } & \multicolumn{2}{|c|}{ Publication Bias } \\
\hline & & & $12(\%)$ & $\mathrm{PH}$ & OR & $95 \% \mathrm{Cl}$ & Ztest & POR & PBeggs & PEggers \\
\hline \multicolumn{11}{|l|}{$655 A>G$} \\
\hline \multirow[t]{5}{*}{ Overall $(n=12)$} & A vs. $G$ & Random & 98.36 & $\leq 0.001$ & 1.101 & $0.638-1.901$ & 0.344 & 0.731 & 0.303 & 0.755 \\
\hline & AA vs. GG & Random & 90.65 & $\leq 0.001$ & 1.562 & $0.919-2.655$ & 1.647 & 0.099 & 0.350 & 0.547 \\
\hline & AG vs. GG & Random & 87.70 & $\leq 0.001$ & 1.493 & $1.147-1.944$ & 2.979 & 0.003 & 0.086 & 0.054 \\
\hline & $A A+A G$ vs. GG & Random & 73.65 & $\leq 0.001$ & 1.298 & $1.085-1.553$ & 2.853 & 0.004 & 0.146 & 0.021 \\
\hline & $A A$ vs. $A G+G G$ & Fixed & 19.33 & 0.260 & 1.150 & $1.020-1.297$ & 2.289 & 0.022 & 0.640 & 0.414 \\
\hline \multicolumn{11}{|l|}{ By ethnicity } \\
\hline \multirow[t]{5}{*}{ Caucasians $(n=6)$} & A vs. $G$ & Random & 99.15 & $\leq 0.001$ & 0.713 & $0.335-1.518$ & -0.878 & 0.380 & 0.452 & 0.645 \\
\hline & AA vs. GG & Random & 75.85 & 0.001 & 1.036 & $0.770-1.394$ & 0.235 & 0.814 & 1.000 & 0.690 \\
\hline & AG vs. GG & Random & 64.92 & 0.014 & 1.079 & $0.931-1.251$ & 1.015 & 0.310 & 0.452 & 0.191 \\
\hline & $A A+A G$ vs. GG & Random & 66.83 & 0.010 & 1.086 & $0.940-1.255$ & 1.177 & 0.264 & 0.707 & 0.280 \\
\hline & $A A$ vs. $A G+G G$ & Fixed & 0.00 & 0.420 & 1.095 & $0.964-1.243$ & 1.397 & 0.162 & 0.452 & 0.573 \\
\hline \multirow[t]{5}{*}{ Asians $(n=4)$} & A vs. $G$ & Fixed & 7.33 & 0.356 & 2.251 & $1.758-2.884$ & 6.425 & 0.00 & 0.734 & 0.381 \\
\hline & AA vs. GG & Fixed & 12.08 & 0.321 & 10.262 & $6.419-16.405$ & 9.727 & 0.00 & 0.296 & 0.282 \\
\hline & AG vs. GG & Random & 88.34 & $\leq 0.001$ & 2.793 & $0.794-9.818$ & 1.601 & 0.109 & 0.734 & 0.226 \\
\hline & $A A+A G$ vs. GG & Fixed & 39.39 & 0.175 & 2.411 & 1.663-3.495 & 4.644 & 0.00 & 0.734 & 0.352 \\
\hline & $A A$ vs. $A G+G G$ & Fixed & 0.00 & 0.760 & 1.660 & $1.155-2.385$ & 2.736 & 0.006 & 1.000 & 0.762 \\
\hline \multicolumn{11}{|l|}{$\begin{array}{l}\text { By Popula- } \\
\text { tion-Based }\end{array}$} \\
\hline \multirow[t]{5}{*}{$\mathrm{PB}(\mathrm{n}=7)$} & A vs. G & Random & 98.79 & $\leq 0.001$ & 0.807 & $0.411-1.586$ & -0.622 & 0.534 & 0.107 & 0.797 \\
\hline & AA vs. GG & Random & 61.27 & 0.017 & 0.960 & $0.738-1.248$ & -0.306 & 0.760 & 0.548 & 0.392 \\
\hline & AG vs. GG & Fixed & 0.799 & 0.423 & 1.037 & $0.959-1.121$ & 0.901 & 0.367 & 0.265 & 0.150 \\
\hline & $A A+A G$ vs. GG & Fixed & 0.00 & 0.479 & 1.047 & $0.971-1.127$ & 1.198 & 0.231 & 0.386 & 0.265 \\
\hline & $A A$ vs. $A G+G G$ & Fixed & 0.00 & 0.677 & 1.081 & $0.950-1.230$ & 1.182 & 0.237 & 0.367 & 0.365 \\
\hline \multirow[t]{5}{*}{$\mathrm{HB}(\mathrm{n}=4)$} & A vs. $G$ & Fixed & 0.00 & 0.591 & 2.153 & $1.763-2.628$ & 7.536 & $\leq 0.001$ & 0.734 & 0.530 \\
\hline & AA vs. GG & Random & 74.01 & 0.009 & 5.873 & $1.911-18.04$ & 3.090 & 0.002 & 0.734 & 0.794 \\
\hline & AG vs. GG & Random & 89.30 & $\leq 0.001$ & 2.955 & $1.111-7.859$ & 2.171 & 0.030 & 0.734 & 0.414 \\
\hline & $A A+A G$ vs. GG & Fixed & 0.00 & 0.415 & 2.513 & $1.876-3.367$ & 6.176 & $\leq 0.001$ & 0.308 & 0.166 \\
\hline & $A A$ vs. $A G+G G$ & Fixed & 0.00 & 0.531 & 1.671 & $1.216-2.297$ & 3.168 & 0.002 & 0.734 & 0.145 \\
\hline \multicolumn{11}{|c|}{ By Genotyping Technique } \\
\hline \multirow[t]{5}{*}{ PCR-RFLP $(n=4)$} & A vs. G & Random & 92.46 & $\leq 0.001$ & 1.725 & $1.038-2.866$ & 2.103 & 0.036 & 1.000 & 0.158 \\
\hline & AA vs. GG & Random & 95.47 & $\leq 0.001$ & 3.821 & $0.963-15.152$ & 1.907 & 0.057 & 0.734 & 0.454 \\
\hline & AG vs. GG & Random & 96.27 & $\leq 0.001$ & 2.556 & $0.828-7.892$ & 1.632 & 0.103 & 0.734 & 0.213 \\
\hline & $A A+A G$ vs. GG & Random & 90.74 & $\leq 0.001$ & 1.961 & $0.999-3.847$ & 1.958 & 0.050 & 1.000 & 0.083 \\
\hline & $A A$ vs. $A G+G G$ & Fixed & 21.29 & 0.283 & 1.366 & $1.133-1.647$ & 3.273 & 0.001 & 0.308 & 0.127 \\
\hline \multirow[t]{5}{*}{ TaqMan $(n=3)$} & A vs. $G$ & Fixed & 0.00 & 0.874 & 0.955 & $0.853-1.069$ & -0.797 & 0.425 & 1.000 & 0.876 \\
\hline & AA vs. GG & Random & 77.78 & 0.011 & 0.660 & $0.285-1.530$ & -0.968 & 0.333 & 1.000 & 0.789 \\
\hline & AG vs. GG & Fixed & 0.00 & 0.742 & 0.952 & $0.816-1.110$ & -0.633 & 0.527 & 0.296 & 0.261 \\
\hline & $A A+A G$ vs. GG & Fixed & 0.00 & 0.902 & 0.945 & 0.816-1.094 & -0.760 & 0.447 & 0.296 & 0.037 \\
\hline & $A A$ vs. $A G+G G$ & Fixed & 0.00 & 0.484 & 0.950 & $0.732-1.231$ & -0.390 & 0.696 & 1.000 & 0.780 \\
\hline \multicolumn{11}{|l|}{$1151 T>A$} \\
\hline \multirow[t]{5}{*}{ Overall $(n=8)$} & A vs. $G$ & Fixed & 41.92 & 0.099 & 2.462 & $2.350-1.635$ & 3.378 & $\leq 0.001$ & 0.063 & 0.013 \\
\hline & AA vs. GG & Fixed & 0.00 & 0.535 & 3.189 & $0.1-30.756$ & 1.003 & 0.316 & NA & NA \\
\hline & AG vs. GG & Fixed & 38.80 & 0.121 & 2.416 & 1.669-3.496 & 4.678 & $\leq 0.001$ & 0.063 & 0.011 \\
\hline & $A A+A G$ vs. GG & Fixed & 41.31 & 0.103 & 2.654 & $1.610-4.375$ & 3.828 & $\leq 0.001$ & 0.220 & 0.055 \\
\hline & $A A$ vs. $A G+G G$ & Fixed & 0.00 & 0.546 & 2.990 & $0.310-28.834$ & 0.947 & 0.343 & NA & NA \\
\hline
\end{tabular}


CRC risk was observed in Asians for $-93 \mathrm{G}>\mathrm{A}$ and $655 \mathrm{~A}>\mathrm{G}$ polymorphisms, but not in Caucasians. It should be considered that the apparent inconsistency of these results may underlie differences in population background, source of controls, lifestyle, disease prevalence, sample size, and also by chance as well as possible limitations due to the relatively small sample size. The current available data support the multifactorial nature of $\mathrm{CRC}$, and both genetic and environmental factors play an important role in development of CRC. Thus, it is unlikely that the same gene polymorphisms may play different roles in cancer susceptibility, because cancer is a complicated multi-genetic disease, and different genetic backgrounds may contribute to the discrepancy.

Present meta-analysis results were not consistent with a previous meta-analysis on MLH1 -93G $>$ A and $655 \mathrm{~A}>\mathrm{G}$ polymorphisms with CRC risk. ${ }^{51,52}$ In 2012, Wang et al. included six case-control studies with 17,791 cases and 13,782 controls on MLH1 $-93 \mathrm{G}>\mathrm{A}$ polymorphism. Their results suggested that MLH1 -93G $>$ A polymorphism was associated with increased risk of CRC under the heterozygote $(\mathrm{OR}=$ $1.06,95 \% \mathrm{CI}=1.01-1.11)$, and the dominant $(\mathrm{OR}=1.06$, $95 \% \mathrm{CI}=1.01-1.11)$ models. ${ }^{51}$ In the more recently meta-analysis, Chen et al. included 13 case-control studies on hMLH1 -93G $>$ A, nine studies on $655 \mathrm{~A}>\mathrm{G}$, and seven studies on 1151T $>$ A. They have reported that there is a significant association between hMLH1 1151T $>$ A polymorphism and CRC risk, but not with hMLH1 655A $>\mathrm{G}$ and $-93 \mathrm{G}>\mathrm{A}$ polymorphisms. Additionally, they have found similar results by subgroup analyses according to quality score and genotyping methods. ${ }^{52}$ However, their meta-analysis might be generated conflicting results, which had insufficient power in the meta-analysis because the number of studies was considerably smaller than that needed for the achievement of robust conclusions. In addition, due to small size meta-analysis, they could not rule out the possibility of publication bias. With more studies about hMLH1 polymorphisms and CRC have available recently, our updated meta-analysis, which has the largest sample size thus reported, we found that the $655 \mathrm{~A}>\mathrm{G}$ and $1151 \mathrm{~T}>\mathrm{A}$ polymorphisms were associated with risk of CRC. Moreover, we found that they wrongly calculated HWE test for both cases and controls in their meta-analysis. Therefore, cumulative meta-analyses have suggested that no significant association was observed between hMLH1 polymorphisms and CRC, as evidence accumulated by time.
Heterogeneity is a potential problem when interpreting the results for most meta-analyses, and finding out the sources of heterogeneity is one of important goals of meta-analyses ${ }^{.53-55}$ In the present meta-analysis there was obvious between-study heterogeneity. ${ }^{54,55}$ Three subgroup analyses were conducted by ethnicity, control source, and smoking status and the heterogeneity still existed. Despite some diversity in the studies about designs, sample sizes, inclusion criteria, and ethnicity, significant heterogeneity between studies was only observed for the $-93 \mathrm{G}>\mathrm{A}$ and $655 \mathrm{~A}>\mathrm{G}$ polymorphism. Thus, we performed subgroup analyses by ethnicities, genotyping methods and source of controls to explore the sources of heterogeneity. The results showed that the heterogeneity disappeared or decreased in several subgroups but remained in other subgroups, suggesting that other covariates might confound the association.

The limitations of this meta-analysis should not be ignored when interpreting the results. First, the meta-analysis was limited by the relatively small number of eligible studies for $1151 \mathrm{~T}>\mathrm{A}$ polymorphism, which may fail to provide enough statistical power to detect a possible or weak effect of the polymorphism on CRC and limited our ability to perform subgroup analyses. Second, only articles published in English or Chinese were selected, potentially causing a language bias. Third, our analysis was limited to Asian and Caucasian ethnicities, and it is uncertain whether these results are generalizable to other ethnicities. Forth, there was significant between-study heterogeneity for two genes in the overall and Caucasians. In addition, the unknown factors including lifestyles and environments may account for the heterogeneity in study results and the lack of significant findings in the overall and Caucasian populations. Fifth, some studies were hospital-based, while others were population-based. Thus, selection bias might exist. Finally, CRC is a multifactorial disease that results from complex interactions between various genes and environmental factors. Our results were based on unadjusted estimates; data were not stratified by other main confounding variables such as age, gender, lifestyle, diet, major systemic illness etc., because sufficient information was not available from those studies.

In summary, the study inconsistent with the previous meta-analyses suggested that hMLH1 -93G $>$ A and $1151 \mathrm{~T}>\mathrm{A}$ polymorphisms may be associated with 
the risk of CRC. Moreover, the $-93 \mathrm{G}>\mathrm{A}$ polymorphism is associated with the susceptibility of CRC in Asian population. However, to ascertain a definitive conclusion on hMLH1 1151T >A polymorphism, well-designed epidemiologic studies with larger sample size and more ethnic groups are suggested to fur- ther clarify the association. Moreover, gene-gene and gene-environment interactions studies should also be considered in future studies.

\section{Conflict of interest}

The authors declare no conflict of interest.

\section{RESUMO}

OBJETIVO: Tem havido crescente interesse no estudo da associação entre polimorfismos do gene mutL homólogo 1 humano (hMLH1) e risco de câncer colorretal (CRC). No entanto, os resultados de estudos anteriores não são conclusivos. Assim, uma meta-análise foi conduzida para obter uma estimativa mais precisa dos efeitos desse gene.

MÉTODOs: Uma pesquisa abrangente foi realizada nas bases de dados PubMed, Embase, Chinese Biomedical Literature até 70 de janeiro de 2018. Odds ratio (OR) com 95\% de intervalo de confiança (IC) foi utilizado para avaliar a força da associação.

RESULTADOS: Finalmente, foram identificados 38 estudos de casos e controles em 32 publicações, atendendo aos nossos critérios de inclusão. Houve 14 estudos com 20.668 casos e 19.533 controles em hMLH1-93G>A, 11 estudos com 5.786 casos e 8.867 controles em 655A>G e cinco estudos com 1.409 casos e 1.637 controles em 1151T>Um polimorfismo. Os resultados combinados mostraram que os polimorfismos 655A>G e 1151T>A estavam significativamente associados ao risco de $C R C$, enquanto que o polimorfismo -93G>A não estava significativamente associado ao risco de CRC. Quanto à etnia, os polimorfismos de -93G $>$ A e 655A>G foram associados ao risco aumentado de $C R C$ entre os asiáticos, mas não entre os caucasianos. Mais interessante, a análise de subgrupos indicou que 655A>G pode aumentar o risco de CRC em subgrupos PCR-RFLP e HB.

CONCLUSÃO: Inconsistente com a meta-análise anterior, esta meta-análise mostra que os polimorfismos hMLH1655A>G e 1151T>A podem ser fatores de risco para CRC. Além disso, o polimorfismo -93G>A está associado à susceptibilidade do CRC na população asiática.

PALAVRAS-CHAVE: Câncer colorretal. hMLH1. Polimorfismo. Meta-análise.

\section{REFERENCES}

1. Khoram-Abadi KM, Forat-Yazdi M, Kheirandish S, Saeidi N, Zarezade Z, Mehrabi N, et al. Asian Pac J Cancer Prev. 2016;17(6):301520.

2. Forat-Yazdi $M$, Gholi-Nataj $M$, Neamatzadeh $H$, Nourbakhsh $P$ Shaker-Ardakani H. Association of XRCC1 Arg399Gln Polymorphism with Colorectal Cancer Risk: A Huge Meta-Analysis of 35 Studies. Asian Pac J Cancer Prev. 2015;16(8):3285-91.

3. Namazi A, Abedinzadeh M, Nourbaksh P, Neamatzadeh H. Association between the XRCC3 Thr241Met polymorphism and risk of colorectal cancer: a meta-analysis of 5,193 cases and 6,645 controls. Asian Pac Cancer Prev. 2015;16(6):2263-8.

4. Jemal A, Siegel R, Xu J, Ward E. Cancer statistics. CA Cancer J Clin. 2010;60(5):277-300

5. Xie M, Zhao F, Zou X, Jin S, Xiong S. The association between CCND1 G870A polymorphism and colorectal cancer risk: A meta-analysis. Medicine (Baltimore). 2017;96(42):e8269.

6. Wang L, Qian J, Ying C, Zhuang Y, Shang X, Xu F. X-ray cross-complementing groups 1 rs 1799782 C>T polymorphisms and colorectal cancer susceptibility: A meta-analysis based on Chinese Han population. J Cancer Res Ther. 2016;12(Supplement):C264-C267.

7. Kheirelseid EA, Miller N, Chang KH, Curran C, Hennessey E, Sheehan M, et al. Mismatch repair protein expression in colorectal cancer. | Gastrointest Oncol. 2013;4(4):397-408.

8. Hsieh P, Yamane K. DNA mismatch repair: Molecular mechanism, cancer, and ageing. Mech Ageing Dev. 2008;129(7-8):391-407.

9. Broustas CG, Lieberman HB. DNA Damage Response Genes and the Development of Cancer Metastasis. Radiat Res. 2014;181(2):111-130.

10. Ricker CN, Hanna DL, Peng C, Nguyen NT, Stern MC, Schmit SL, et al. DNA mismatch repair deficiency and hereditary syndromes in Latino patients with colorectal cancer. Cancer. 2017;123(19):3732-3743.

11. Ellison AR, Lofing J, Bitter GA. Human MutL homolog (MLH1) function in DNA mismatch repair: a prospective screen for missense mutations in the ATPase domain. Nucleic Acids Res. 2004;32(18):5321-5338.
12. Peltomäki $P$. Role of DNA mismatch repair defects in the pathogenesis of human cancer. J Clin Oncol. 2003;21(6):1174-9.

13. Aarnio M, Sankila R, Pukkala E, Salovaara R, Aaltonen LA, de la Chapelle $A$, et al. Cancer risk in mutation carriers of DNA-mismatch-repair genes. Int J Cancer. 1999; 81(2):214-218.

14. Millar AL, Pal T, Madlensky L, Sherman C, Temple L, Mitri A, et al. Mismatch repair gene defects contribute to the genetic basis of double primary cancers of the colorectum and endometrium. Hum Mol Genet. 1999; 8(5):823-829.

15. Ito E, Yanagisawa $Y$, Iwahashi $Y$, Suzuki $Y$, Nagasaki H, Akiyama $Y$, et al. A core promoter and a frequent single-nucleotide polymorphism of the mismatch repair gene hMLH1. BiochemBiophys Res Commun. 1999;256(3):488-494.

16. Shin $\mathrm{KH}$, Shin $\mathrm{H}$, Kim JH, Park JG. Mutational analysis of promoters of mismatch repair genes hMSH2 and hMLH1 in hereditary nonpolyposis colorectal cancer and early onset colorectal cancer patients: identification of three novel germ-line mutations in promoter of the hMSH2 gene. Cancer Res. 2002;62(1):38-42.

17. Raptis S, Mrkonjic M, Green RC, Pethe VV, Monga N, Chan YM, et al. MLH1 -93G>A promoter polymorphism and the risk of microsatellite-unstable colorectal cancer. J Natl Cancer Inst. 2007;99(6):463-474.

18. Chen H, Taylor NP, Sotamaa KM, Mutch DG, Powell MA, Schmidt AP, et al. Evidence for heritable predisposition to epigenetic silencing of MLH1. Int J Cancer. 2007;120(8):1684-1688.

19. Tulupova E, Kumar R, Hanova M, Slyskova J, Pardini B, Polakova V, et al. Do polymorphisms and haplotypes of mismatch repair genes modulate risk of sporadic colorectal cancer? Mutat Resch. 2008;648(1-2):40-45.

20. Samowitz WS, Curtin K, Wolff RK, Albertsen H, Sweeney C, Caan B], et al. The MLH1 -93 G>A promoter polymorphism and genetic and epigenetic alterations in colon cancer. Genes Chromosomes Cancer. 2008; 47(10):835-844.

21. Koessler $T$, Oestergaard $M Z$, Song $H$, Tyrer J, Perkins B, Dunning AM, et al. Common variants in mismatch repair genes and risk of colorectal cancer. Gut. 2008; 57(8):1097-1101. 
22. Allan JM, Shorto J, Adlard J, Bury J, Coggins R, George R, et al. UK NCRI Colorectal Clinical Studies Group; Colorectal Cancer Study Group. MLH1 -93G>A promoter polymorphism and risk of mismatch repair deficient colorectal cancer. Int J Cancer. 2008;123(10):2456-9.

23. Campbell PT, Curtin K, Ulrich CM, Samowitz WS, Bigler J, Velicer CM, et al. Mismatch repair polymorphisms and risk of colon cancer, tumour microsatellite instability and interactions with lifestyle factors. Gut. 2009;58(5):661-667.

24. van Roon EH, van Puijenbroek M, Middeldorp A, van Eijk R, de Meijer EJ, Erasmus D, et al. Early onset MSI-H colon cancer with MLH1 promoter methylation, is there a genetic predisposition? BMC Cancer. 2010;10:180.

25. Whiffin N, Broderick P, Lubbe SJ, Pittman AM, Penegar S, Chandler I, et al. MLH1-93G $>$ A is a risk factor for MSI colorectal cancer. Carcinogenesis. 2011;32(8):1157-1161.

26. Savio AJ, Lemire M, Mrkonjic M, Gallinger S, Zanke BW, Hudson T], et al. $M L H 1$ region polymorphisms show a significant association with $C p G$ island shore methylation in a large cohort of healthy individuals. PLoS One. 2012;7(12):e51531.

27. Muniz-Mendoza R, Ayala-Madrigal ML, Partida-Perez M, Peregrina-Sandoval J, Leal-Ugarte E, Macias-Gomez N, et al. MLH1 and XRCC1 polymorphisms in Mexican patients with colorectal cancer. Genet Mol Res. 2012; 11(3):2315-2320.

28. Nizam ZM, Abdul Aziz AA, Kaur G, Abu Hassan MR, Mohd Sidek AS, Lee YY, et al. Contribution of the MLH1 -93G>a promoter polymorphism in modulating susceptibility risk in Malaysian colorectal cancer patients. Asian Pac J Cancer Prev. 2013;14(2):619-624.

29. Martinez-Uruena N, Macias L, Perez-Cabornero L, Infante M, Lastra E, Cruz II, et al. Incidence of $-93 \mathrm{MLH1}$ promoter polymorphism in familial and sporadic colorectal cancer. Colorectal Dis. 2013; 15(3):e118-123.

30. Djansugurova L, Zhunussova G, Khussainova E, Iksan O, Afonin G, Kaidarova D, et al. Association of DCC, MLH1, GSTT1, GSTM1, and TP53 gene polymorphisms with colorectal cancer in Kazakhstan. Tumour Biol. 2015;36(1):279-289.

31. Li G, Hu F, Yuan F, Fan J, Yu Z, Wu Z, et al. Intronic and promoter polymorphisms of $\mathrm{hMLH} 1 / \mathrm{hMSH} 2$ and colorectal cancer risk in Heilongjiang Province of China. J Cancer Res Clin Oncol. 2015;141(8):1393-404.

32. Zhang LL, Tang XJ, Wang XY, Zhu YW, Peng XB, Gong L. A promoter polymorphism in the hMLH1 gene (-93G/A) associated with sporadic colorectal cancer. Oncol Lett. 2016; 12(5):4035-4040.

33. Mik M, Dziki L, Malinowska K, Trzcinski R, Majsterek I, Dziki A. Polymorphism of MSH2 Gly322Asp and MLH1 -93G>A in non-familial colon cancer - a case-controlled study. Arch Med Sci. 2017;13(6):12951302

34. Kim JC, Roh SA, Koo KH, Ka IH, Kim HC, Yu CS, et al. Genotyping possible polymorphic variants of human mismatch repair genes in healthy Korean individuals and sporadic colorectal cancer patients. Fam Cancer. 2004;3(2):129-137.

35. Mei Q, Yan HL, Ding FX, Xue G, Huang JJ, Wang YZ, et al. Single-nucleotide polymorphisms of mismatch repair genes in healthy Chinese individuals and sporadic colorectal cancer patients. Cancer Genet Cytogenet. 2006; 171(1):17-23.

36. Berndt SI, Platz EA, Fallin MD, Thuita LW, Hoffman SC, Helzlsouer KI. Mismatch repair polymorphisms and the risk of colorectal cancer. Int J Cancer. 2007;120(7):1548-1554.

37. Christensen LL, Madsen BE, Wikman FP, Wiuf C, Koed K, Tjonneland A et al. The association between genetic variants in $\mathrm{hMLH} 1$ and $\mathrm{hMSH} 2$ and the development of sporadic colorectal cancer in the Danish population. BMC Med Genet. 2008:9:52.
38. Nejda N, Iglesias D, Moreno Azcoita M, Medina Arana V, Gonzalez-Aguilera J], et al. A MLH1 polymorphism that increases cancer risk is associated with better outcome in sporadic colorectal cancer. Cancer Genet Cytogenet. 2009;193(2):71-77.

39. Picelli S, Zajac P, Zhou XL, Edler D, Lenander C, Dalen J, et al. Common variants in human CRC genes as low-risk alleles. Eur I Cancer. 2010;46(6):1041-1048

40. Milanizadeh S, Khanyaghma M, Haghighi MM, Mohebbi S, Damavand B, Almasi S, et al. Molecular analysis of imperative polymorphisms of MLH1 gene in sporadic colorectal cancer. Cancer Biomark. 2013;13(6):427-432.

41. Peng HX, Xu X, Yang R, Chu YM, Yang DM, Xu Y, et al.Molecular analysis of MLH1 variants in Chinese sporadic colorectal cancer patients. Genet Mol Res. 2016;15(2).

42. Wang Y, Friedl W, Propping P, Li J, Li Z, Wang J. Val384Asp in hMLH1 gene in Chinese, lapanese and German and its etiological role in colorectal cancer. Zhonghua Yi Xue Yi Chuan Xue Za Zhi. 1998;15(5):263-6.

43. Wang Y, Zhou J, Li Z, Wang J, Li J, Gao C, et al. One of the etiological factors of digestive tract cancers in Chinese: the missense mutation Val- 384Asp in the hMLH1 gene. Zhonghua Yi Xue Yi Chuan Xue Za Zhi. 2000;17(2):82-86.

44. Zhang XM, Li JT, Zhu M, Wu XL, Gao P, Zhou P, et al. Study on the relationship between genetic polymorphism Val384Asp in hMLH1 gene and the risk of four different carcinomas. [Article in Chinese]. Zhonghua Liu Xing Bing Xue Za Zhi. 2004;25(11):978-981.

45. Ohsawa T, Sahara T, Muramatsu S, Nishimura Y, Yathuoka T, Tanaka Y, et al. Colorectal cancer susceptibility associated with the hMLH1 V384D variant. Mol Med Rep. 2009;2(6):887-891.

46. Wang $D$, Song $L$, Zhang X. Etiological role of Val384Asp in $\mathrm{hMLH1}$ gene in familial colorectal cancer. Acta Univ Med Nanjing. 2010; 1:1-6.

47. Higgins JPT, Thompson SG. Quantifying heterogeneity in a meta-analysis. Stat Med. 2002;21(11):1539-58.

48. DerSimonian R, Laird N. Meta-analysis in clinical trials. Control Clin Trials. 1986;7(3):177-188

49. Egger M, Davey SG, Schneider M, Minder C. Bias in meta-analysis detected by a simple, graphical test. BMJ. 1997;315(7109):629-634.

50. Begg CB, Mazumdar M. Operating characteristics of a rank correlation test for publication bias. Biometrics. 1994;50(4):1088-1101.

51. Wang T, Liu Y, Sima L, Shi L, Wang Z, Ni C, et al. Association between MLH1 -93G>a polymorphism and risk of colorectal cancer. PLoS One. 2012;7(11):e50449.

52. Chen H, Shen Z, Hu Y, Xiao Q, Bei D, Shen X, et al. Association between MutL homolog 1 polymorphisms and the risk of colorectal cancer: a meta-analysis. J Cancer Res Clin Oncol. 2015 Dec;141(12):2147-58.

53. Sobhan MR, Mehdinejad M, Jamaladini MH, Mazaheri M, Zare-Shehneh $M$, Neamatzadeh $\mathrm{H}$. Association between aspartic acid repeat polymorphism of the asporin gene and risk of knee osteoarthritis: A systematic review and meta-analysis. Acta Orthop Traumatol Turc. 2017;51(5):409-415.

54. Mehdinejad M, Sobhan MR, Mazaheri M, Zare-Shehneh M, Neamatzadeh $\mathrm{H}$, et al. Genetic Association between ERCC2, NBN, RAD51 Gene Variants and Osteosarcoma Risk: a Systematic Review and Meta-Analysis. Asian Pac J Cancer Prev. 2017;18(5):1315-1321.

55. Sobhan MR, Forat-Yazdi M, Mazaheri M, Zare-Shehneh M, Neamatzadeh $\mathrm{H}$. Association between the DNA Repair Gene XRCC3 rs861539 Polymorphism and Risk of Osteosarcoma: a Systematic Review and Meta-Analysis. Asian Pac | Cancer Prev. 2017;18(2):549-555. 